Raghavan N, Varghese K., Mahalingam A., Delhi VSK. (2018). "Simulation Exercise for Collaborative Planning System/ Last Planner System ${ }^{\mathrm{TM}}$ (COLPLASSE) In: Proc. $26^{\text {th }}$ Annual Conference of the International. Group for Lean Construction (IGLC), González, V.A. (ed.), Chennai, India, pp. 1002-1012. DOI: doi.org/10.24928/2018/0429.Available at: www.iglc.net.

\title{
SIMULATION EXERCISE FOR COLLABORATIVE PLANNING SYSTEM / LAST PLANNER SYSTEM ${ }^{\text {TM }}$ (COLPLASSE)
}

\author{
Raghavan $\mathrm{N}^{1}$, Koshy Varghese ${ }^{2}$, Ashwin Mahalingam ${ }^{3}$ and Venkata S.K. Delhi ${ }^{4}$
}

\begin{abstract}
The Last Planner System (LPS ${ }^{\mathrm{TM}}$ )is becoming popular for project management all over the world. Though the practitioners are able to follow the concepts quite well, they sometimes find it difficult to develop the required processes and templates, particularly in organisations and environments where systematic planning practices are not that prevalent. Structured templates, simulations, or games for LPS are also not freely available in the open domain. A simulation exercise, COLPLASSE (COLlaborative PLAnning System Simulation Exercise), has been developed to cater to this felt need.

COLPLASSE is based on simple Excel spreadsheets and uses work plans for developing Look Ahead Plans and Weekly planning over the many weeks required for project completion. It has provisions to simulate random delays due to inclement external environmental conditions or variations in productivity due to various causes. It computes PPC automatically and simultaneously draws continuous charts for PPC and Root Cause Analysis over the Project completion period. It is simple to use and with further improvements being planned, can develop into a powerful tool for training or simulation or actual use along with LPS. Further research is proposed to be done using this simulation with various groups to evaluate its capabilities for helping early practitioners to use LPS.
\end{abstract}

\section{KEYWORDS}

Collaborative Planning System, Simulation, Excel spreadsheet, Work plans template

1 Professor of Practice, Dept. of Civil Engineering, Indian Institute of Technology Madras, Chennai, India nraghavan@iitm.ac.in

2 Professor, Dept. of Civil Engineering, Indian institute of Technology Madras, Chennai, India koshy@iitm.ac.in

3 Associate Professor, Dept. of Civil Engineering, Indian Institute of Technology Madras, Chennai, India, mash@iitm.ac.in

4 Assistant Professor, Civil Engineering Dept., Indian Institute of Technology Bombay, Mumbai, India, venkatad@iitb.ac.in 


\section{INTRODUCTION}

\section{USE OF PLANNING TOOLS}

The Last Planner System ${ }^{\text {TM }}$ (LPS) (Ballard 1994) has been a significant game-changer in the construction arena and a dramatic innovation or a fresh breath of air in the humdrum planning domains, improving considerably the certainty levels, flow of work and consistency in performance. Though it has been around for a long time, nascent practitioners, particularly organisations with little track record for systematic planning as in developing countries, took quite a bit of time to develop the processes required for its optimum usage in the field. For construction personnel steeped in the usage of CPM schedules, the processes required for developing and using Phase schedules, Look Ahead Plans (LAPs), measurement systems such as Plan Percent Completed (PPC) or Root Cause Analysis (RCA) appear to be difficult to put into practice in the field. Often coaches have to work with the field personnel to develop templates and schedules for deploying LPS in the projects. For initial training also, the basic concepts of LPS are better understood if templates are used to demonstrate how exactly LAPs, etc have to be developed and used. Over time and across different geographical and industrial arenas LPS TM has been undergoing adaptations during implementation in different circumstances and contexts and Collaborative Planning System (CPS) being practised in India of late, is one such adaptation (Raghavan, et al 2014, Raghavan 2015). CPS brings in larger support to the Last Planners from the Project Manager and Planning Manager with a Big Room approach for the developmental meetings in contexts where the Last Planners are not that well conversant with planning processes and interactive approaches (where planning as a process is itself not that well developed, as in smaller organizations in developing countries). Over time with continuous guidance and support the Last Planners become more proficient but in the earlier stages a Collaborative approach is required.

The constraint of space precludes detailed exposition of the software or describing an example problem in this paper, though they are addressed adequately in the User's Manual for the software.

\section{AVAILABLE SIMULATIONS AND TEMPLATES}

Simulations and games are quite helpful for training in the use of management techniques and would be particularly useful for using Lean construction concepts and applications in projects (Dukovska-Popovska, et al 2008, Loon et al 2015). Though many simulation games are available in the field of Lean Construction management, hardly any training simulation exercise is available to teach and practise the fundamentals of LPS ${ }^{\text {TM }}$ in the open domain. The available games are somewhat complex or take too long to play out and often require an expert coach to play them out with many players. Some spreadsheet applications are available for LPS usage (Ghafari, 2015) but most of them are not directly suitable for training or for those just starting on their Lean journey. A need has been felt to fill this gap and COLPLASSE (COLlaborative PLAnning System Simulation Exercise) is designed to meet this felt need. 
The aeroplane game (Visionary Products, 2015) is good for demonstrating the push-pull interplay and effect of variable batch-sizing. The Parade of Trades game (Tommelein, et al, 1999) is an excellent starter to demonstrate the impact of workflow variability. LEAPCON (Sacks, et al, 2007)) is good for use with multi-storey construction, demonstrating concepts such as pull and work structuring. Villego (Villego, 2014) has been a classic game which covers practically the entire gamut of LPS concepts but is somewhat complex, is in considerable detail and not handy enough for classrooms or quick training sessions. LEBSCO (Gonzalez, et al, 2014 and Gonzalez, et al, 2015), an LPS-based simulation game is also in somewhat high detail and needs many players, but each in limited roles. Make-a-Card, The Silent squares, Flow-building or Pull-building Lean games are other games covering multiple players and situations.

For classroom teaching or teaching a number of planners simultaneously, COLPLASSE would be appropriate as the spreadsheets can be replicated for each participant and each can progress with the simulation in his own way with different probabilistic scenarios thrown their ways for the simulations for the same given problem. It also covers the concepts of milestone planning, Phase scheduling, LAPs, Constraint solving, productivities, PPC calculations, Root Cause analysis, etc. It is planned to make the software available freely on the open domain.

\section{OVERVIEW}

\section{DOCUMENT STRUCTURE}

This Simulation exercise has been designed to teach the basic processes of LPS to the beginners in Lean Construction Management. This open system simulation employs the well-known and popular Microsoft Excel spreadsheets as the base. It unfolds the Lean planning systems and concepts such as Look Ahead Plan, PPC Analysis, Root Cause Analysis, etc over the construction duration in a series of connected spreadsheets. It can help in teaching initial planning as well as planning updates to remediate production losses due to various factors. It has been kept simple, by design, to enable easy adaptations to a variety of problems, for training as well as for real-life cases. It has builtin automated graphics for depicting PPC trends as well as Root Cause Analysis. Further developments are ongoing mainly by deploying more Excel macros to infuse greater flexibility for tackling larger real-life cases. With its open domain architecture, users can freely make adaptations for varying contexts.

\section{WORKING OF COLPLASSE}

\section{BASICS}

An Initial Sheet (Figure 1) lists out the various basic data required for the simulation. This includes the list of activities and for each activity the quantities of work to be done, number of crews available, and average productivity. It is possible to introduce a Period of Inclement Conditions (such as monsoon) by specifying the serial numbers of the weeks when such conditions prevail, which reduce the productivity of all activities performed in such periods. One can also introduce probabilistically-evolved Productivity Modification 
Factors for each of the work weeks, which increase or decrease the quantity of work performed during that week for the specified activities (due to productivity improvement measures or better planning put in place, or due to unforeseen problems or unresolved constraints, respectively). It is possible to assign specific Root Causes (from the available library of typical causes) which cause such reduction in productivity. Contractual milestones can be specified by stipulating the serial numbers of the weeks for completion of key activities. Number of working days per week and number of working hours per day can also be changed. The simulation has as many intermediate spreadsheets as the number of work weeks, for the work plans. The Final Sheet, at the end of the various work weeks, contains the results of analysis of the execution parameters, compiled automatically during the play-out of the various weeks.

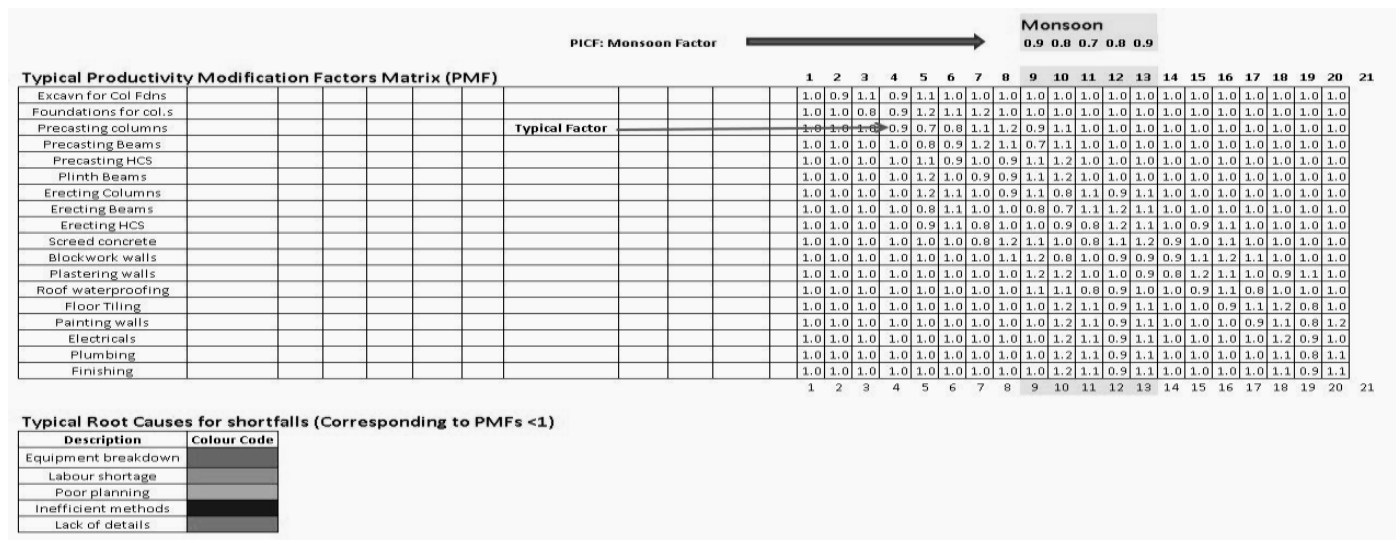

Figure 1: Screen shot of Initial Sheet

Each intermediate worksheet (Figure 2) or work plan covering one week of work depicts a four-week rolling Look Ahead Plan (LAP) as well as the progress in the Current Week (First of the four weeks). Each sheet lists out all the activities of work, the balance quantity of work to be performed for each activity as at the beginning of the Current Week, the basic productivity value assumed for each activity and the number of work teams provided. The actual work performed for any activity in the Current week, which is derived from the above data, is further modified according to Productivity Modification Factors which depend upon the external factors prevailing in the Current Week as well as on any exceptional situations occurring in this week. This progression is carried forward week after week until project completion. 


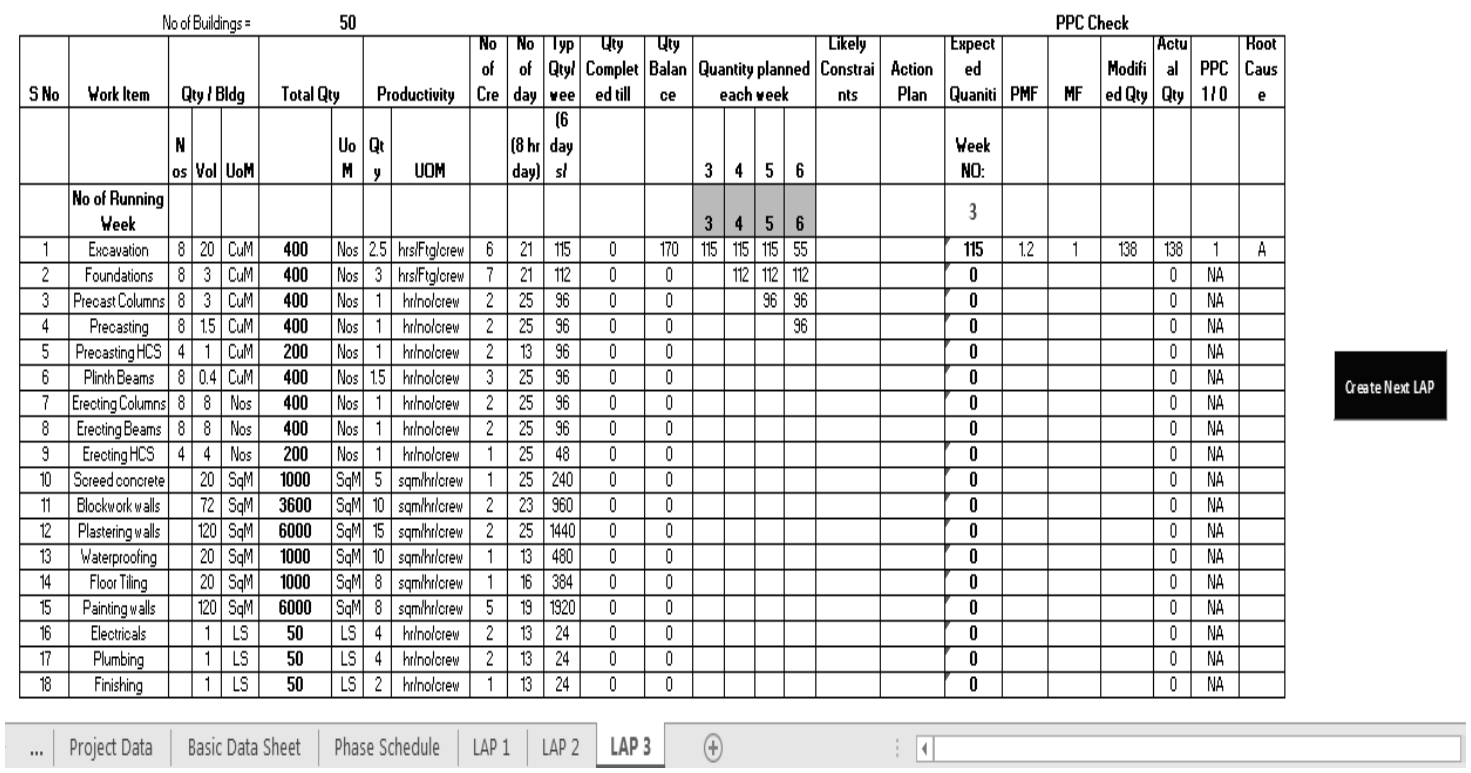

Figure 2: Screen shot of typical Running Sheet for the example problem.

Initially, a Milestone Schedule and Phase Schedules have to be prepared, forming the basis for the LAPs. The Expected Quantity planned for any week for any activity can be adjusted by changing the number of crews assigned to suit completion of milestones as per the current Phase Schedule, which should again be in conformity with the overall Milestone Schedule drawn up initially.

\section{GLOSSARY OF KEYWORDS}

LAP - Look-Ahead Plan, typically covering 4 weeks

Current Week - Serial Number of the first of the 4 weeks in the current LAP

PICF - Period of Inclement Conditions Factor (for instance, due to monsoon)

PMF - Productivity Modification Factor $(<1$ if there are problems for execution of that activity during that week because ofconstraints; can also be $>1$ iffavourable conditions prevail))

RC - Root Cause responsible leading to PMF being less than 1

No. of Hours worked/week $=$ Standard hours per day $x$ no. of days/ week $(=6 \times 8=48$ hours in this example)

Balance Quantity = Quantity remaining to be executed as of the beginning of the Current Week for any activity

Possible Quantity = Productivity x No. of Crews x No. of hours worked per week

Expected Quantity = Quantity assigned to the first of the 4 weeks in the LAP Modified Quantity = Expected Quantity x PICF x PMF

Actual Quantity = Modified Quantity or Balance Quantity, whichever is less.

Score for Activity $=$ Equal to 1 if Actual Quantity $>=$ Expected Quantity, Else $=0$

PPC for Current week = No. of "1"s for Activity Scores / Total no. of Activities executed during current Week x100 


\section{WORK QUANTITY MODIFICATION FACTORS}

The Initial or the basic Spreadsheet lists all the activities required for the construction work along with the quantities of work for each activity, number of crews envisaged and the average productivity of work for each activity, all of which can be used to determine the number of weeks required for completion of each activity. The Possible Quantity of work which can be done in a week for any activity is the product of the Productivity, the number of crews and the number of hours worked per week. For meeting intermediate milestones, the Possible Quantities can be adjusted by modifying the number of crews. For purposes of this simulation, the planner does not have any control over the basic productivity data; the average productivity specified initially gets modified by the factor due to Inclement Conditions and the Productivity Modification Factor which can decrease or increase the productivity.

A concept of a Period of Inclement Conditions (PIC) has been specified covering a few weeks in between during which climatic or social or economic conditions which hamper good construction progress could prevail. For instance, in the example problem which covers 20 weeks and 18 activities, monsoon conditions prevail over five specified weeks. The fall in production due to reduction in working hours during such a period is simulated by a PIC Factor (PICF) which is less than 1, which reduces the Possible Quantity of work calculated as mentioned above. In the example problem during the five weeks of monsoon PICF is specified as $0.9,0.8,0.7,0.8$ and 0.9 for the five weeks, to simulate progressively deepening and easing monsoon conditions over the five weeks.

The potential problems which may arise due to various glitches such as equipment breakdown, wrong methods, bad planning, etc are taken care of by assigning a Productivity Modification Factor (PMF). The PMF numbers are assigned by the random number generation algorithm of Excel. They are in the range of 0.7 to 1.2 in this example problem, thus decreasing the productivity sometimes (when less than 1.0) and increasing it (when greater than 1.0) at some other times. This range is set in the Initial Sheet and is modifiable. The final quantity of work executed would then be equal to the original Possible Quantity as worked out earlier multiplied by PICF and PMF. For each application of PMF less than 1, a corresponding Root Cause (RC) (generated or picked from a library of Root Causes) would be assigned for the affected activity, for the users to get a flavour of what all things can go wrong during execution. A pie chart which shows the relative prevalence of the various Root Causes at any given time is built up automatically, week after week.

\section{THE STRUCTURE OF SPREADSHEETS}

There are essentially three spreadsheet systems in this tool: Basic Data, Running Plans and Analysis Sheet. Basic Data given in the Initial Sheet comprises the list of various items of work and their respective quantities as well as typical productivities for execution, etc. The PICF matrix and RC library would be found here. Initially depending on the milestones specified, the Planner has to develop Phase Schedules (typically 1 to 3 phases for this example, which has five milestones) covering all the activities and conforming to the set Milestones. 
Fig. 2 shows a typical Running Sheet for an example problem, covering various work items right from excavation for foundations to finishing work. The respective quantities for the various work items involved are given in the Initial Sheet. It also gives the expected productivities (inverse of productivity in some cases) for the various work items and number of crews available for each work. It also indicates the weekly schedule template, overall milestones, and scope of work for the three Phases involved and monsoon months. Milestones are specified for Completion of excavations and foundations, Completion of all pre-casting, Completion of all concreting work, and Completion of all finishing except services (electrical, plumbing) and Final Completion.

The Running Plan is a spreadsheet for the Current Week and is also a Look-Ahead Plan (LAP) covering four weeks starting with the Current Week, with all the activities listed along with their original quantities as well as the Balance Quantity as of the beginning of the Current Week. There will be as many Running Plan sheets as the number of work weeks. For each week the Balance Work quantity for each activity will be automatically taken as the Balance Quantity of the previous week less the actual quantity executed during the previous week. In each Running Plan, the Planner has to assign quantities for the 4 weeks of the LAP to the activities which need to be executed in this four- week period as per the relevant Phase Schedule and duly considering the prevailing PICF. However, these quantities will have to be less than or equal to the Possible Quantity, which is derived from productivity, number of crews and number of working days per week. If more quantity of work is desired to be executed, then the number of crews will have to be increased. The Expected (Current Week) Quantities will then be automatically assigned from the first week of the four-week LAP. Then once the Current Week number is entered in the designated Cell, the relevant PMFs will appear automatically for the activities which are being executed in the Current week, having been generated in a random fashion but within the overall specified range given in the Initial Sheet (0.7 to 1.2 in this example). The Modified Quantity column will show the above-mentioned Expected Quantity duly modified by PICF and PMF. Since the PMF can sometimes be more than 1.0, the Modified Quantity may come out to be more than the Balance Quantity and in such a case the Actual Quantity column will show the lesser of Modified Quantity or Balance Quantity. If the Actual Quantity is greater than or equal to the Expected Quantity, then PPC for that activity will be automatically shown as 1; else it will be shown as 0. If PICF is $<1$ then the Root Cause Type column will indicate a RC Type Number taken from the Root Cause library, corresponding to the specified PICF. In another variation, the RC can be picked out from the library of potential RCs.

Once all the relevant activities are operated upon as above, the weekly PPC will be automatically calculated and the PPC Chart shown graphically at the bottom (Figure 3) will be automatically updated including the Current Week's data. Similarly, the Root Causes Pie Chart shown will also get automatically updated considering the Current Week's data. Columns are also provided to indicate Constraints and Acton Plans to solve the Constraints for the various activities in the LAP to give a realistic flavour during training. 


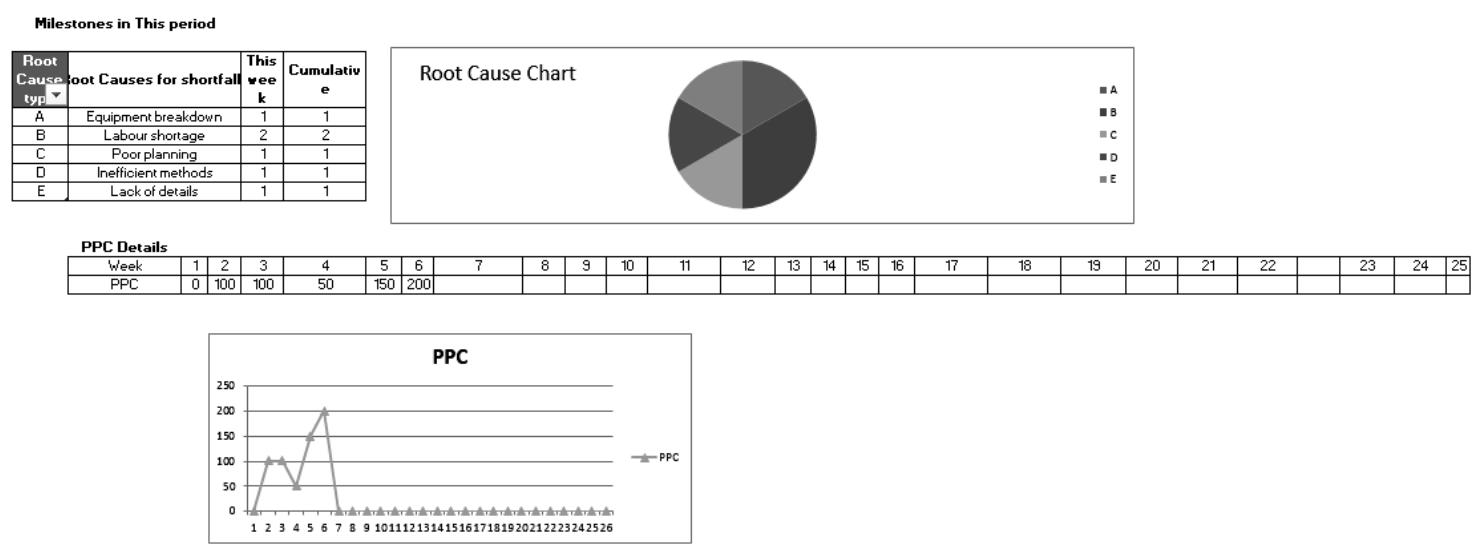

Figure 3: Screen shot of automated Root Causes Pie Chart and PPC chart

Thereafter the Current Running Sheet is copied using macros to a fresh sheet and renamed for the next week's serial number. The above procedure is then repeated again and again until the completion of all activities is reached.

Then the Analysis sheet at the end of the Excel file will be automatically updated compiling the data from all the work weeks and will show the following: the overall PPC variation chart, overall average PPC, the overall Root Causes Pie Chart and the variation in the number of crews deployed in all the weeks for the various activities (as a measure of cost). The quantities executed for the various items in the various weeks as well as the PICF and PMF for the various weeks are also shown in a compiled form.

\section{USING COLPLASSE}

In a classroom exercise, each learner can have one copy on his laptop/ computer and run the simulation over the duration of the project. Either the example project (given with fully developed data along with the software) can be used or the instructor can develop a new project model. The various users will get different scenarios because of the randomness of the PMFs and may choose a different number of crews at different times. The Final Sheet compiles the project history and different attempts can be compared for their efficiencies of execution. Using the sum of the products of the number of crews deployed and their standard productivities over the weeks as the base maximum possible output, the actual quantity executed will indicate the efficiency of operations. If the exercise is played out in a group assigning various roles to different people, the identification of the Constraints and their resolution can be done in a collaborative manner, exemplifying the Last Planner process.

\section{Another Method of playing out the Simulation}

- Representatives are required for the 6 major trades to interact together: Foundations (excavation, foundations \& plinth beams), Concrete Structures (pre-casting \& erection, screed concrete), Walls (block work, plastering \& painting), Finishing (Waterproofing, tiling, and overall finishing), Electricals, and Plumbing. 
- One overall Planning Manager for coordination and one Project Manager for overall management will also be identified. The Planning Manager will prepare all the Schedules and Charts, based on inputs from all concerned.

- The Team will first prepare (together in a Big Room exercise) Phase Schedules for the three phases [Phase I - Excavation, foundations \& pre-casting; Phase II - Basic Structure; Phase III- Finishing (plastering, painting, waterproofing, tiling, services, completion], compatible with the stipulated Milestones, by using a Pull system.

- Then they will prepare together LAPs (Look Ahead Plans) for 4 weeks at a time by Pull system, duly considering the Monsoon period. The likely constraints and action plans will also be identified. The Monsoon season has been identified and during this season there will be a reduction in productivity and a Monsoon Factor $(\mathrm{PICF}<1)$ is given for each Monsoon week to reduce the Possible Quantity of work which can be done. PMFs with varying values will also be specified for the various weeks and should be duly taken into account. The number of crews can be increased in weeks when additional production is desired to meet specified milestones.

- Finally, the actual completion period should be compared with the theoretical completion period. The Root Cause Analysis should also be compiled and discussed. The variation in number of crews will give an idea of the costs involved.

Alternatively, the instructor can demonstrate a typical execution to a class with the excel sheets projected on a screen for all to see.

In further versions to be developed for actual usage, project practitioners may be able to use COLPLASSE for actual LAPs and keeping track of weekly progress.

\section{LIMITATIONS AND POSSIBLE FUTURE WORK}

An attempt has been made to reduce complexity without sacrificing authenticity for this Simulation. In the current phase of development, COLPLASSE can be used mainly for the simpler problems; daily planning has to be done off-line and integrated with weekly plan; constraints listing and resolution have to be done off-line and integrated. It is also slightly tedious to operate over several weeks for simulation projects as each time the Running Plan spreadsheet has to be copied over from the previous Week (though done automatically using a macro) and modified for the Current Week as required. However, for actual projects when only one Running Plan will be active at any given time for the Current Week it may not be a constraint. In the next phase of development, more Excel sheet macros can be used to simulate more processes. It should also be possible to expand the number of weeks for the LAPs from 4 to 8 weeks.

\section{DISCUSSION AND CONCLUSIONS}

An example problem covering 20 work weeks and 18 activities has been given along with all required basic data. Students and trainees can practice with the example problem to get a good feel of the LPS process. Actual practitioners can also input data from their projects and run COLPLASSE week after week to automate the templates for easy 
operations. The data for the example problem as well as screenshots of typical worksheets are given in Fig.s 1 and 2.

The COLPLASSE simulation would be quite useful for beginners of Lean practices to understand the basic procedures of actual LPS implementation and provide them with templates for monitoring LPS implementation. For the serious practitioners, COLPLASSE can provide a simulation tool for evaluating different methods of executing a project. The simulation tool has invoked good interest from Lean practitioners in India to whom this was exposed. It is hoped that the larger Lean community will also find it to be of good interest. COLPLASSE would be freely available for downloading from a specified domain for open usage, while duly acknowledging the Developers.

Further research is proposed to be done using this simulation with various groups to evaluate its capabilities for helping nascent practitioners to use LPS. The results of such research will be presented in a future conference.

\section{ACKNOWLEDGEMENTS}

The authors acknowledge with thanks the support given by Mr Anon, M.Tech Student, for his good supporting work in the development of the Excel sheets, and that of Ms Anon, MS Student for her support in developing the Excel Macros.

\section{REFERENCES}

Ballard, G., "The Last Planner" Spring Conference of the Northern California Construction Institute, Monterey, CA, April 1994, 22-14

Dukovska-Popovska, I, Hove-Madsen, V., and Nielsen, K. B., "Teaching lean thinking through game: Some challenges," in 36th European Society for Engineering Education (SEFI) on Quality Assessment, Employability \& Innovation, 2008.

Ghafari, A., 2015. VPlanner®: "The Visual Planning Solution for Lean Project Delivery" [Online] Available at:<http://www.myvplanner.com/vsps/>

Gonzalez, V., Orozco, F., Senior, B, Ingle, J., Forcael, E, Alarcon, LF. -"LEBSCO:LeanBased Simulation Game for Construction Management Classrooms", Jr. of Professional issues in Engineering Education and Practice, Feb., 2015.

Gonzalez,V.A., Senior, B., Orozco, F., Alarcon, L.F., Best, A. - "Simulating Lean Production Principles in Construction: A Last Planner-Driven Game", Proceedings, IGLC-22, June 2014, 1221-1232

Loon, M., Evans, J., Kerridge, C., "Learning with a Strategic Management simulation game: A Case Study", The International Journal of Management Education, 13(2015), 227-236.

Raghavan, N., Kalidindi, S., Mahalingam, A., Varghese, K., and Ayesha, A. (2014). "Implementing Lean Concepts on Indian Construction Sites: Organisational Aspects and Lessons Learned." Proceedings of the 22nd annual conference of the International Group for Lean Construction, Oslo, 1181-1190.

Raghavan, N, "Implementing Lean Concepts in India in Construction Sites - A Trial and its Outcome", Indian Lean Construction Conference ILCC2015, Mumbai, 2015, 39-52 
Sacks, R., Esquenazi, A. and Goldin, M. (2007). "LEAPCON: Simulation of Lean Construction of High-Rise Apartment Buildings", J. of Constr. Engrg. and Mg mt.ASCE, 133 (7) 529-539.

Tommelein, I., Riley, D. and Howell, G (1999). "Parade Game: Impact of Work Flow

Variability on Trade Performance”. J. of Constr. Engrg. and Mgmt.-ASCE, 125 (5) 304310.

Villego (2014). Villego-Last Planner Simulation. (available at:http://www.villego.com/).

Visionary Products (2014). "The Airplane Game". (available athttp://www.visionaryproducts.biz/Home/Products/LZPM/tabid/61/Default.aspx). 